Portland State University

PDXScholar

\title{
Determination of Electronic Cigarette Liquid Water Content by NMR Spectroscopy
}

Michael Lethin

Portland State University

Follow this and additional works at: https://pdxscholar.library.pdx.edu/honorstheses

Let us know how access to this document benefits you.

\section{Recommended Citation}

Lethin, Michael, "Determination of Electronic Cigarette Liquid Water Content by NMR Spectroscopy" (2017). University Honors Theses. Paper 373.

https://doi.org/10.15760/honors.366

This Thesis is brought to you for free and open access. It has been accepted for inclusion in University Honors Theses by an authorized administrator of PDXScholar. Please contact us if we can make this document more accessible: pdxscholar@pdx.edu. 
Determination of Electronic Cigarette Liquid Water Content by NMR Spectroscopy

By

Michael Lethin

An undergraduate honors thesis submitted in partial fulfillment of the requirements for the degree of

Bachelor of Science

in

University Honors

and

Biochemistry

Thesis Advisor

Dr. David Peyton

Portland State University

2017 


\section{Abstract:}

Electronic cigarette liquid (e-liquid) often degrades during vaporization in electronic cigarettes via a number of pathways, including dehydration reactions. The amount of water present in eliquid may influence the number and amount of degradation products formed during the vaporization process. In order to allow a subsequent test of this hypothesis, a method was developed for the quantification of water in e-liquid by way of nuclear magnetic resonance spectroscopy (NMR) peak integration. The method was verified using standard addition, and was found to have a range of accuracy and precision from 0.361 to 0.619 and 0.127 and 0.474 mole-percent respectively $(\mathrm{N}=9)$. 
$\underline{\text { Table of Contents }}$

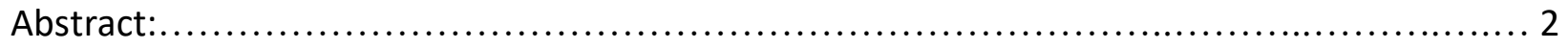

Introduction: ......................................................................... 4

1.1 Electronic Cigarettes: History and Usage: ..........................................4

1.2 Electronic Cigarettes: Form and Function: ........................................ 5

1.3 Degradation: ................................................................... 6

1.4 The Quantification of Water: ................................................. 7

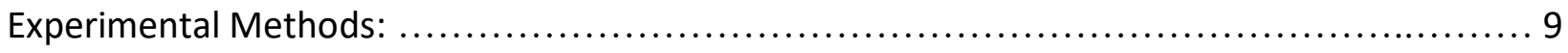

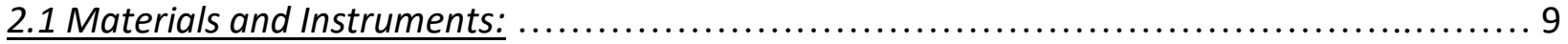

2.2 Methods: ...................................................................... 10

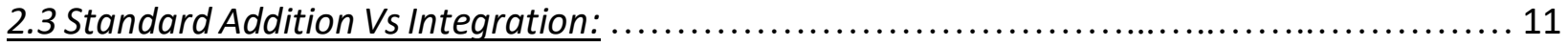

Results and Discussion: ............................................................. 12

Propylene Glycol: ................................................................. 12

Glycerol: ................................................................... 13

Glycerol/Propylene Glycol Mixtures: ............................................... 13

Commercial Electronic Cigarette liquids: .............................................. 14

Discussion: .......................................................................... 14

Conclusion: ................................................................ 15

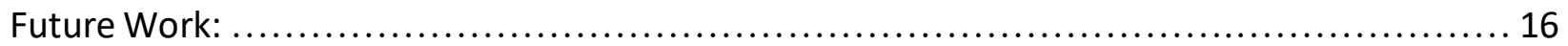

References: ......................................................................... 17

Supplemental Figures:............................................................. 19 
Introduction:

\subsection{Electronic Cigarettes: History and Usage}

The history of the modern electronic cigarette starts with Chinese pharmacist and inventor Hon Lik in $2001^{1}$. After witnessing his father die of lung cancer, Hon Lik personally quit smoking and began researching alternatives to tobacco cigarettes. His first design used a high frequency ultrasound element to vaporize nicotine-containing liquid, much like nebulizer machines used to administer asthma medications ${ }^{2}$. While the device effectively delivered nicotine, the resulting smoke was thin and did not imitate the thick smoke of burning tobacco.

While first introduced to the Chinese market in 2003, the electronic cigarette did not make it to the United States until 2007. By that time, the device was significantly evolved and heating elements had replaced piezoelectric elements as the means of vaporization ${ }^{3}$. A small hobbyist community developed around the device, and over the next several years the market for electronic cigarettes grew. Many online forums developed where users shared their own device modifications and e-liquid recipes ${ }^{4}$. Some of these modifications became standard features on commercial devices, including detachable, rechargeable battery units, and integrated heating element/liquid tank/mouthpiece units called "clearomizers 5 ." As the hobbyist electronic cigarette community grew, so did the popularity of the devices among average consumers.

In the early 2010s, use and public knowledge of electronic cigarettes skyrocketed. Between 2010 and 2013, the percentage of adults aware of electronic cigarettes in the United States nearly doubled from $40.7 \%$ to $79.7 \%$ and the percentage of adults who have used electronic cigarettes more than doubled from $1 \%$ to $2.6 \%{ }^{6}$. Among high school student's electronic cigarette usage rose from $4.5 \%$ in 2013 to $16 \%$ in $2016^{7}$. These trends were mirrored by a rise in 
electronic cigarette advertising spending which also saw a marked increase from \$6.4 million in 2011 to $\$ 115$ million in $2014^{8}$. As these trends will likely continue, a better understanding of the health implications of these devices is needed to inform the public as well as the policy makers that regulate them.

\subsection{Electronic Cigarettes: Form and Function}

Electronic cigarettes are divided into two main categories: disposable electronic cigarettes, and rechargeable, refillable, customizable vaporizers. Popular vaporizer designs today are comprised of a number of components: mouthpiece, tank, atomizer, wick, battery and microcontroller (Figure 1). Most of these parts are detachable from one another and can be replaced and customized by the user. These components can be grouped into two main parts, the clearomizer unit and the battery unit.

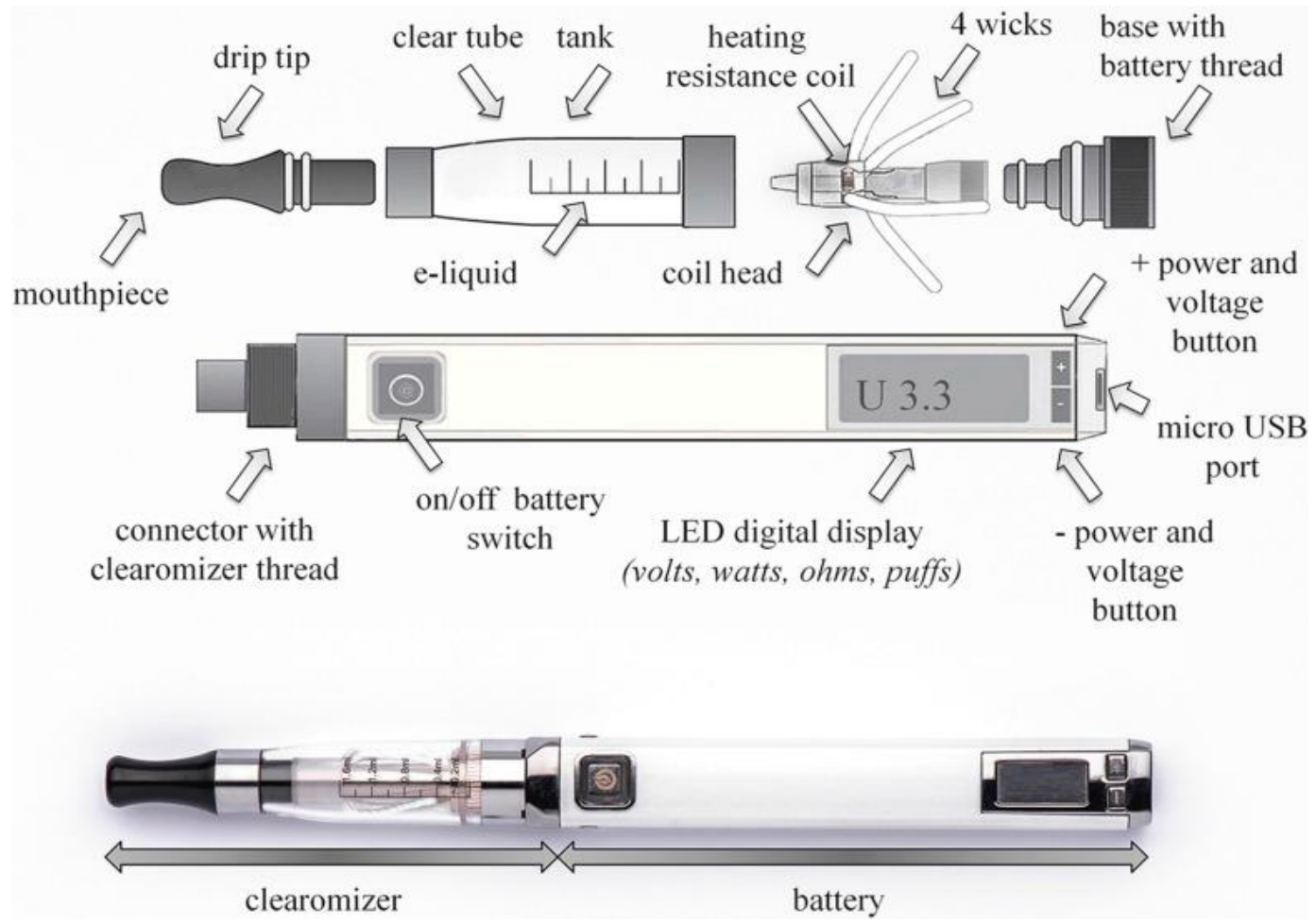

Figure 1. A typical modern vaporizer type electronic cigarette with its components labeled ${ }^{9}$. 
The liquid consumed by electronic cigarettes, commonly referred to as e-liquid, e-juice or vape juice is typically comprised of mostly propylene glycol, glycerol or a mixture of both with various flavorings and sometimes but not always nicotine ${ }^{10}$. Typically, the e-liquid is stored in the tank where it is wicked to the heating element. The heating element then heats the e-liquid to the point that it is aerosolized and inhaled through the mouthpiece by the user. The temperature of the coil can be roughly controlled by altering the wattage delivered by the battery. This can be done by either utilizing the built-in microcontroller or by using heating elements of various resistance values combined with different voltages.

\subsection{Degradation}

The main ingredients in e-liquid, propylene glycol $\left(\mathrm{C}_{3} \mathrm{H}_{8} \mathrm{O}_{2}\right)$ and glycerol $\left(\mathrm{C}_{3} \mathrm{H}_{8} \mathrm{O}_{3}\right)$ are relatively common in consumer products. In the food, beverage, and personal care industries, glycerol is commonly used as a sweetener, moistener, and volume additive in many hand soaps, moisturizers, and shampoos. While propylene glycol is primarily used to manufacture plastics, it is also a common food additive and anti-freeze substitute. In their liquid state, both compounds are relatively non-toxic and unreactive ${ }^{11,12}$. Things change however, when they are heated together (or independently) in the atomizers of electronic cigarettes.

During the aerosolization process, propylene glycol and glycerol can react with each other and themselves as well as flavorants, sweeteners, nicotine, and atmospheric oxygen to form a wide variety of organic compounds, dubbed "degradation compounds ${ }^{13}$." To analyze these

compounds, previous studies ${ }^{14,15}$ used mixtures of only propylene glycol and glycerol as simplified analogs to e-liquid. These analogs were aerosolized using electronic cigarettes, and 
the aerosol was analyzed to identify the major compounds and the chemical reaction pathways that produced them. Two primary reaction pathways for the degradation of propylene glycol and glycerol that have been identified are oxidation and dehydration ${ }^{14,15}$. Oxidation through either free atmospheric $\mathrm{O}_{2}$ or the $\mathrm{C}-\mathrm{H}$ bonds of other molecules can lead to the production of hydroxyacetone, lactaldehyde and glyceraldehyde ${ }^{14}$. Thermal dehydration was shown to generate glycidol, 3-hydroxypropanal, acetone, allyl alcohol, and propanal -- among others ${ }^{14}$.

Dehydration reactions (e.g. Figure 2) are responsible for a large portion of the degradation products detected in electronic cigarette vapor. Applying Le Chatelier's Principle to these reactions it can be hypothesized that the concentration of water present in the e-liquid at the time of aerosolization affects the rate of reaction. Changes to the rate of reaction therefore change the amount of degradation present in the inhaled aerosol. To test this hypothesis, a method that reliably and accurately measures the concentration of water in the starting eliquid is needed.

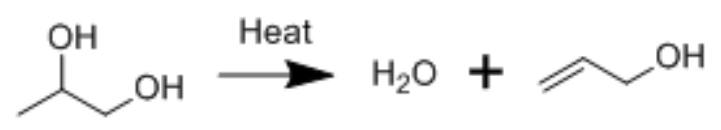

Figure 2. Propylene glycol becomes allyl alcohol via a dehydration reaction ${ }^{16}$.

\subsection{The Quantification of Water}

Traditionally, water content in solvents has been quantified via specialized gas chromatography mass spectroscopy (GCMS) or Karl Fischer Titration ${ }^{17,18}$. Both of these methods require expensive, specialized equipment and training outside the scope of what might normally be available in a typical university research laboratory, and can degrade the sample 
through heating or exposure to chemical reagents. The present study looks to expand the use of nuclear magnetic resonance spectroscopy (NMR), a commonly available analytical tool, to analyze electronic cigarette e-liquids for their water content. NMR has often been employed in the identification and quantification of organic compounds (such as those found in electronic cigarette liquid and vapor) and is non-destructive, meaning that it does not chemically alter the sample. However, traditional methods of NMR have struggled to quantify water due to the broad shape of the water peak typically found in organic solvents and the dependence of this water peak's chemical shift on concentration ${ }^{19}$.

Traditionally, NMR is performed using a very high ratio of solvent to sample, typically involving 1-10 $\mu \mathrm{L}$ of sample in $600 \mu \mathrm{L}$ of deuterated NMR solvent. This produces a highlydetailed spectrum that can be useful when attempting to identify individual compounds that could be obscured due to the wider peaks that result from high concentration samples. When the traditional high solvent ratio technique is tested with mixtures of propylene glycol/glycerol/water in the solvent DMSO- $d_{6}$, the water peak overlaps with propylene glycol and glycerol peaks, making integration of the individual water peak difficult. To remedy this, it was proposed that the ratio of sample to solvent be altered in an attempt to shift the water peak to a location in the spectrum where it could be more easily integrated. A ratio of $20 \%$ sample to $80 \%$ solvent was shown to consistently shift the water peak to a more advantageous position, where this overlap was no longer an issue.

While NMR peak integration is an established method for quantifying concentrations of chemical compounds in a sample, verification of the accuracy of the technique in specific 
scenarios is recommended. Chemical reactions between NMR solvent, water, propylene glycol and glycerol in their liquid state are unlikely to occur at room temperature and alter the observed spectra. However, extensive hydrogen bonding between these compounds can still significantly affect NMR spectra by shifting peaks. This study aims to verify that simple water peak integration using a 20:80 sample:solvent ratio is a valid method of quantifying the water content of propylene glycol and/or glycerol e-liquid analogs despite these potential peak shifts.

\section{Experimental Methods:}

\subsection{Materials and Instrumentation}

Deionized water was obtained from Science Building 1 at Portland State University and further distilled using a Corning AG-1b still. USP grade propylene glycol $\left(\mathrm{C}_{3} \mathrm{H}_{8} \mathrm{O}_{2}\right)$, glycerol $\left(\mathrm{C}_{3} \mathrm{H}_{3} \mathrm{O}_{3}\right)$, were obtained from Sigma-Aldrich (St. Louis, $\mathrm{MO}$ ) and used to create e-liquid analogs. Dimethyl sulfoxide- $d_{6}(D, 99.9 \%)+0.05 \%$ V/V TMS, spectrometric grade, was obtained from Cambridge Isotope Laboratories (Tewksbury, MA) and used as the NMR solvent. All NMR data was collected using a Bruker Ultrashield Avance-III $600 \mathrm{MHz}$ NMR spectrometer with a BBO probe and the ' $\mathrm{zg} 30$ ' pulse sequence with the following modified parameters; 64 scans, a D1 relaxation of 3 seconds, and a rotation speed of $20 \mathrm{~Hz}$. The FIDs were recorded using the associated software, Topspin, and analyzed using MestReNova 9.1 NMR software. Microsoft Excel was then used to compile the data. 


\subsection{Methods}

Aliquots of water (10 $\mu \mathrm{L}$ each) were titrated four times into samples containing $80 \%$ DMSO- $d_{6}$ and $20 \%$ USP grade propylene glycol (PG) and/or glycerol (GY) by volume. After each addition, the samples were evaluated using onedimensional proton NMR spectroscopy (the zg30 pulse sequence) with a 3-second relaxation delay period. The PG and GY peaks present in the NMR spectrum for each sample were integrated, and the precision and accuracy of these integrations were calculated. The sample to solvent ratio with the best compromise between peak width and integration value precision (20:80 sample:solvent by volume) was then used in a series of standard addition and integration. First, $480 \mu \mathrm{L}$ of DMSO- $d_{6}$ was tested as a blank. A $120 \mu \mathrm{L}$ sample of PG was added to the initial $480 \mu \mathrm{L}$ of

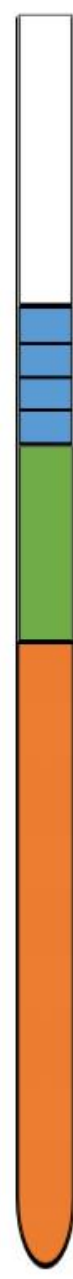

Water Additions $-4 \times 10 \mu \mathrm{L}$ Sample $-120 \mu \mathrm{L}$ DMSO- $d_{6}-480 \mu \mathrm{L}$
Figure 3. The composition of a typical SA sample after 4 water additions. ${ }^{20}$ DMSO- $d_{6}$, inverted 3 times and mixed between each inversion by a VWR Vortexer 2 , and then evaluated by NMR. This was followed by the independent addition of 4 aliquots of water (10 $\mu \mathrm{L}$ each) with mixing and testing after each addition (Figure 3). The water content of the blank was subtracted from each of the sample spectra using the TMS peak as a reference, and the remaining water peaks were integrated with respect to the doublet at $1.02 \mathrm{ppm}$, which arises from the 3 methyl protons on PG ( $\gamma$ position in Figure 4). The standard addition method was then used to calculate the amount of water originally present in the sample. The peak 
integration values of the sample before water addition were also used to independently calculate water content using the integration method, discussed in the following section. Trials were performed in triplicate, and the calculated values from each trial were compared to calculate the accuracy and precision for both methods. The same method was then applied to samples of GY, where the water peak was integrated with respect to the doublet of quintuplets at $3.37 \mathrm{ppm}$, which represents 4 protons from glycerol ( $\alpha$ position in Figure 4). Samples of a 50:50 mole percent mixture of PG:GY, as well as various commercial e-liquids, were also tested and the water peak integrated with respect to the PG doublet at $1.02 \mathrm{ppm}$.

\subsection{Standard Addition vs Integration}

In the standard addition method, the integration values were plotted as a function of the volume of water added to the sample vs the water peak integration values. The slope of the plotted points was extrapolated using the trend line function of Microsoft Excel (SF 1). The $Y$
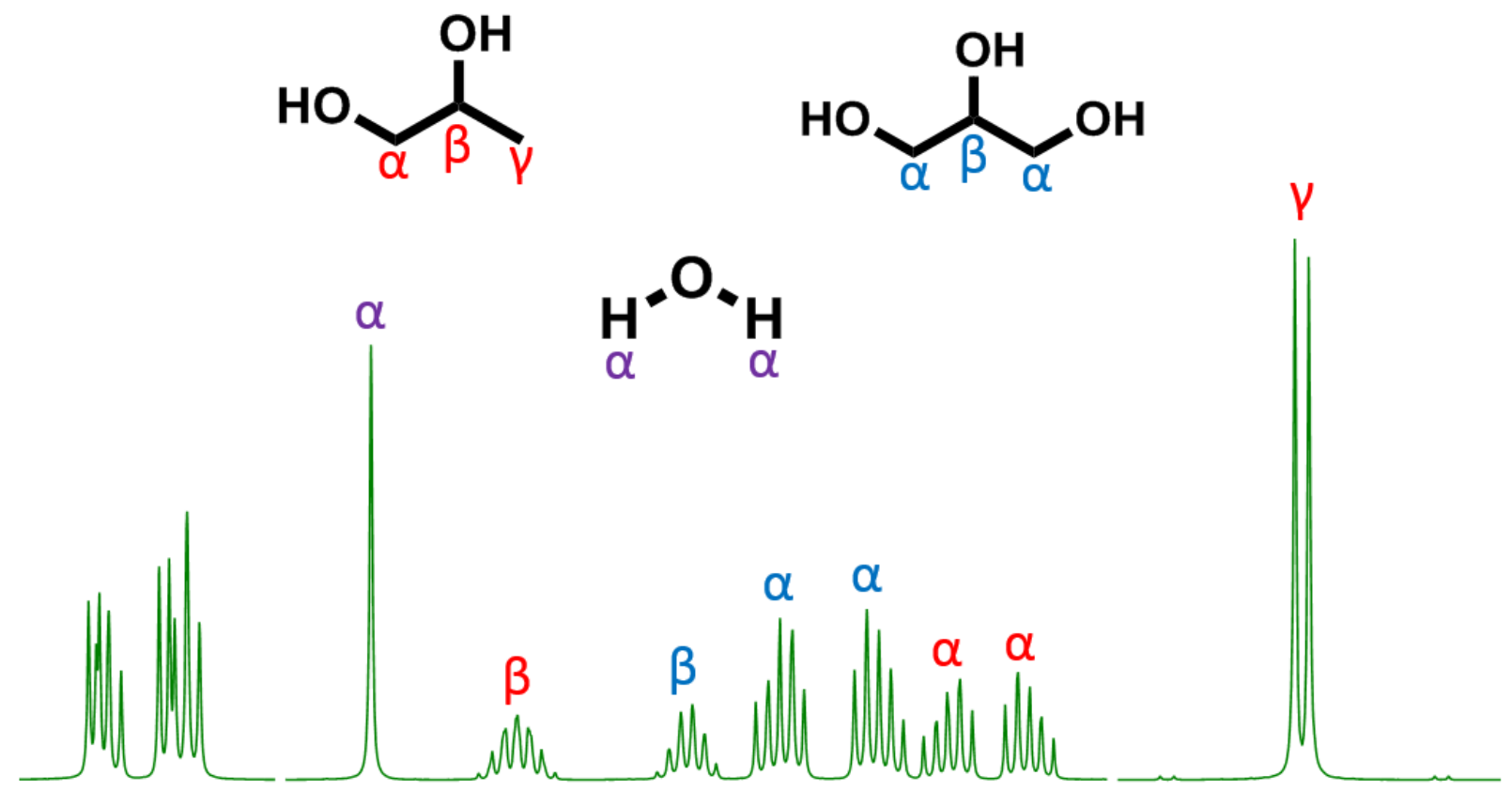

Figure 4. NMR spectrum of a 50/50 mixture of propylene glycol and glycerol with labeled peaks, $40 \mu \mathrm{L} \mathrm{H}_{2} \mathrm{O}$ added ${ }^{20}$ 
intercept was then divided by the slope to find the $\mathrm{X}$ intercept, which is the volume of water originally present in the sample. This can then be converted into total moles and mole percentage relative to the PG and/or GY. In the integration method, the peak integration values were divided by the number of protons represented by the peak. These values represented the molar quantities of each compound present in the sample. The molar quantities were then added together to produce the total number of moles, and each individual ratio was divided by the total to give mole percentage.

$\underline{\text { Results and Discussion: }}$

\subsection{Propylene Glycol}

Line graphs for the three standard additions of water to propylene glycol were created by plotting the water peak integration area vs 10,20 , 30 , and $40 \mu \mathrm{L}$ volume of water added to the sample
Table 1. Propylene Glycol Standard Addition and Integration Method Mole \% data.

\begin{tabular}{|c|c|c|}
\hline & SA Mole \% & Integration Mole \% \\
\hline Trial 1 & 0.525 & 0.44 \\
\hline Trial 2 & 0.313 & 1.10 \\
\hline Trial 3 & 0.133 & 1.12 \\
\hline AVE & 0.323 & 0.887 \\
\hline SD & 0.196 & 0.387 \\
\hline
\end{tabular}

and then averaged (SF 1). The extrapolated molar water contents for each sample were $0.525 \%$, $0.313 \%$, and $0.013 \%$ (Table 1) each with $R^{2}$ values of 0.9999 (SF 1, SF 2, and SF 3). The average molar water content of the PG samples acquired from the same bottle was $0.323 \%$ with a standard deviation of $0.196 \%(\mathrm{~N}=3)$. Via the integration method, trials 1,2 , and 3 were found to have molar contents of $0.44 \%, 1.1 \%$, and $1.12 \%$ respectively with an average of $0.887 \%$ and a standard deviation of $0.387 \%(\mathrm{~N}=3)$. The difference between the averages of the two methods was $0.564 \%$. 


\subsection{Glycerol}

The same procedure performed on samples

Table 2. Glycerol Standard Addition and Integration Method Mole \% data.

of glycerol resulted in extrapolated water molar

contents of $2.82 \%, 2.73 \%$, and $3.55 \%$ (Table 2 ) with

$R^{2}$ values of $0.9992,0.9999$, and 0.9995

respectively (SF 4, SF 5, and SF 6). The average

\begin{tabular}{|c|c|c|}
\hline & SA Mole \% & Integration Mole \% \\
\hline Trial 1 & 2.82 & 3.12 \\
\hline Trial 2 & 2.73 & 4.6 \\
\hline Trial 3 & 3.55 & 3.67 \\
\hline AVE & 3.03 & 3.80 \\
\hline SD & 0.45 & 0.75 \\
\hline
\end{tabular}

molar water content was $3.03 \%$ with a standard deviation of $0.45 \%(\mathrm{~N}=3)$. Using the standard

integration method, molar contents of $3.12 \%, 4.6 \%$, and $3.67 \%$ were calculated, with an

average of $3.79 \%$ and standard deviation of $0.963 \%(\mathrm{~N}=3)$. The difference between the averages of the two methods was $0.75 \%$.

\subsection{Glycerol + Propylene Glycol Mixtures}

The procedure was performed again, this time on samples comprised of 50 mole\% glycerol and 50 mole\% propylene glycol. The standard addition method resulted in molar water percentages of $0.86 \%, 0.83 \%$, and $1.89 \%$ (Table 3 )
Table 3. Glycerol/Propylene Glycol 50/50 mixture $\mathrm{SA}$ and Integration Method data.

\begin{tabular}{|c|c|c|}
\hline & SA Mole $\%$ & Integration Mole \% \\
\hline Trial 1 & 0.87 & 1.34 \\
\hline Trial 2 & 0.83 & 1.15 \\
\hline Trial 3 & 1.89 & 1.31 \\
\hline AVE & 1.20 & 1.27 \\
\hline SD & 0.60 & 0.10 \\
\hline
\end{tabular}

with $R^{2}$ values of $0.9998,0.9997$, and 0.9998 respectively (SF 7, SF 8 , and SF). The average molar content was $1.2 \%$ with a standard deviation of $0.60 \%(\mathrm{~N}=3)$. Using the integration method, molar water content of $1.34 \%, 1.15 \%$, and $1.31 \%$ were calculated with an average of $1.27 \%$ and a standard deviation of $0.1 \%(\mathrm{~N}=3)$. The difference in average molar water content between the two methods was $0.46 \%$. 


\subsection{Commercial Electronic cigarette liquids}

The same two methods were performed on single trials of commercial electronic cigarette liquids, with only the Halo brand
Table 4. Commercial Electronic Cigarette Liquid SA and Integration Method data

\begin{tabular}{|c|c|c|c|}
\hline & Standard Addition & Integration & Difference \\
\hline Atmos "Tobacco" & 23.824 & 24.391 & 0.567 \\
\hline Atmos "Menthol" & 21.326 & 20.19 & 1.136 \\
\hline Halo "Café Mocha" & 26.912 & 27.23 & 0.318 \\
\hline
\end{tabular}

"Café Mocha" e-liquid containing nicotine $(6 \mathrm{mg} / \mathrm{mL})$. Atmos brand "Tobacco" flavored e-liquid was determined to have a molar water content of $23.82 \%$ (Table 4 ) via the standard addition method, with an $\mathrm{R}^{2}$ value of 0.9985 (SF 10). The integration method resulted in a molar content of $24.39 \%$, with a $0.57 \%$ difference between methods. Atmos brand "Menthol" flavored e-liquid was determined to have a molar content of water of $21.33 \%$ via the standard addition method with an $R^{2}$ value of 1 (SF 11). The integration method found a molar content of $20.19 \%$, resulting in a 1.14\% difference. Halo brand "Café Mocha" flavored e-liquid was determined to have a molar water content of $26.91 \%$ via the standard addition method with an $\mathrm{R}^{2}$ value of 0.9997 (SF 12). The integration method resulted in a molar content of $26.91 \%$, a difference of $0.32 \%$.

\subsection{Discussion}

The goal of this study was to test the viability of using a modified integration method with a 20:80 sample:solvent ratio to quantify the amount of water present in electronic cigarette liquid. The three sets of trials using propylene glycol, glycerol, and a mixture of the two showed that the integration method reliably results in water content values within $1 \%$ of 
the standard integration method, under the $2 \%$ inaccuracy that is considered the acceptable limit for quantitative $\mathrm{NMR}^{21}$. One potential limitation of this study is what could be perceived as an unusually large standard deviation within the data set. In most cases, standard deviations that are a large percentage of the value measured are cause for concern. However, in this case, the standard deviations can be explained by the very small amount of water being measured in the samples. While the standard deviations are indeed at times a large percentage of the amount of water measured, it likely that if the amount of water in the sample was scaled to 10 to 15 times the amount in this study, the standard deviations would stay on the same scale. Confidence in this outcome comes from the extremely consistent $R^{2}$ values found in the standard addition experimental data.

The data presented in this study therefore suggests that the integration method can be used as an inexpensive and non-destructive alternative to Carl-Fischer Titration and GCMS for water content determinations. It is accurate and precise enough to quantify water in solutions of propylene glycol and glycerol, which are analogous to electronic cigarette liquid. The trials of commercial e-liquid also show that in most cases measurements of molar concentration via the integration method come within $1 \%$ of the standard integrations method and always within at least $2 \%$.

\section{Conclusion:}

A novel and simple method for the quantification of water concentrations in electronic cigarette liquid using 1D proton NMR has been developed. This method uses an 80:20 solvent:sample ratio to aid in the consistent integration of the target water peak. This method 
will be useful in the continued study of electronic cigarette vapor decomposition products, by allowing connections to be made between decomposition of electronic cigarette vapor and water content of e-liquid.

Future Work:

This method will be particularly useful in the study of the effect of water on the decomposition of electronic cigarette liquid while vaping. Since dehydration reactions are one of the primary pathways for propylene glycol and glycerol decomposition, the presence of a large percentage of water in the liquid during vaporization could significantly alter the equilibrium of these reactions. This, along with boiling point depression effects have the potential to reduce the number and severity of degradation products present in electronic cigarette vapor. Preliminary research performed by Anna Duell and myself at Portland State University show just such results ${ }^{22}$, with increased concentrations of water trending towards decreased decomposition. Further research is needed to characterize these trends in order to better inform the public of any possible risks related to electronic cigarette use. 


\section{$\underline{\text { References }}$}

[1] Sridi, N. (2013, October 18). "I was sure that the electronic cigarette would be welcomed with open arms". https://www.sciencesetavenir.fr/sante/i-was-sure-that-the-electroniccigarette-would-be-welcomed-with-open-arms 26020

[2] Lik, H. (2007). U.S. Patent No. US2007267031. Washington, DC: U.S. Patent and Trademark Office.

[3] Bhatnagar, A., Whitsel, L., \& Ribisl, K. et al. (2014). Electronic Cigarettes: A Policy Statement by the American Heart Association. Circulation, 130(16), 1418-1436.

[4] Grothaus, M. (2016, July 14). Trading addictions: the inside story of the e-cig modding scene. https://www.engadget.com/2014/10/01/inside-story-e-cig-modding-uk/

[5] Denham, A. (2013, June 09). Brothers who took a punt on a new market.

[6] King, B., Patel, R., \& Nguyen, K. et al. (2014). Rends in Awareness and Use of Electronic Cigarettes among U.S. Adults, 2010-2013. Nicotine \& Tobacco Research.

[7] U.S. Department of Health and Human Services (2016). E-Cigarette Use Among Youth and Young Adults: A Report of the Surgeon General. National Library of Medicine Cataloging-in-Publication Data.

[8] E-cigarette Ads and Youth. (2016, January 05). Retrieved from https://www.cdc.gov/vitalsigns/ecigarette-ads/

[9] Giroud, C., Cesare, M. D., Berthet, A., et al. (2015). E-Cigarettes: A Review of New Trends in Cannabis Use. International Journal of Environmental Research and Public Health, 12(8), 9988-10008.

[10] Peace, M., Baird T., Smith, N., et al. (2016) Concentration of Nicotine and Glycols in 27 Electronic Cigarette Formulations. Journal of Analytical Toxicology, 40(6), 403-407.

[11] U.S. Food and Drug Administration (2016) Subchapter B - Food for Human Consumption. § 184.1666. Propylene glycol. Code of Federal Regulations, 21-3.

[12] U.S. Food and Drug Administration (2016) Subchapter B - Food for Human Consumption. § 182.1320. Glycerin. Code of Federal Regulations, 21-3.

[13] Flora, J., Meruva, N., \& Huang, C. et al. (2016). Characterization of potential impurities and degradation products in electronic cigarette formulations and aerosols. Regulatory Toxicology and Pharmacology, 74, 1-11.

[14] Jensen, R. P. (2016). Thermal Decomposition of Electronic Cigarette Liquids (Doctoral dissertation). Portland State University. 
[15] Jensen, R. P., Strongin, R. M., \& Peyton, D. H. (2017). Solvent Chemistry in the Electronic Cigarette Reaction Vessel. Scientific Reports, 7, 42549.

[16] Smith, J. G. (2014). Organic chemistry. New York, NY: McGraw-Hill.

[17] Tavčar, E., Turk, E., \& Kreft, S. (2012). Simple Modification of Karl-Fischer Titration Method for Determination of Water Content in Colored Samples. Journal of Analytical Methods in Chemistry, 2012, 1-6.

[18] Zhang, Y., Wang, C., \& Armstrong, D. et al. (2011). Rapid, Efficient Quantification of Water in Solvents and Solvents in Water Using an Ionic Liquid-based GC Column. LCGC Europe, 24(10), 516-252.

[19] Duell, A., Portland State University Department of Chemistry. Peyton Lab. "unpublished data."

[20] Lethin, M., Duell, A., Peyton, D., (2016) Determination of Electronic Cigarette Liquid Water Content by NMR Spectroscopy. Poster presented at 2016 Portland State University ACS Undergraduate Poster Symposium.

[21] Bharti, S., \& Roy, R. (2012). Quantitative ${ }^{1} \mathrm{H}$ NMR spectroscopy. Trends in Analytical Chemistry, 35, 5-26.

[22] Duell, A., Lethin, M., Portland State University Department of Chemistry. Peyton Lab. "unpublished data." 


\section{$\underline{\text { Supplemental Figures }}$}

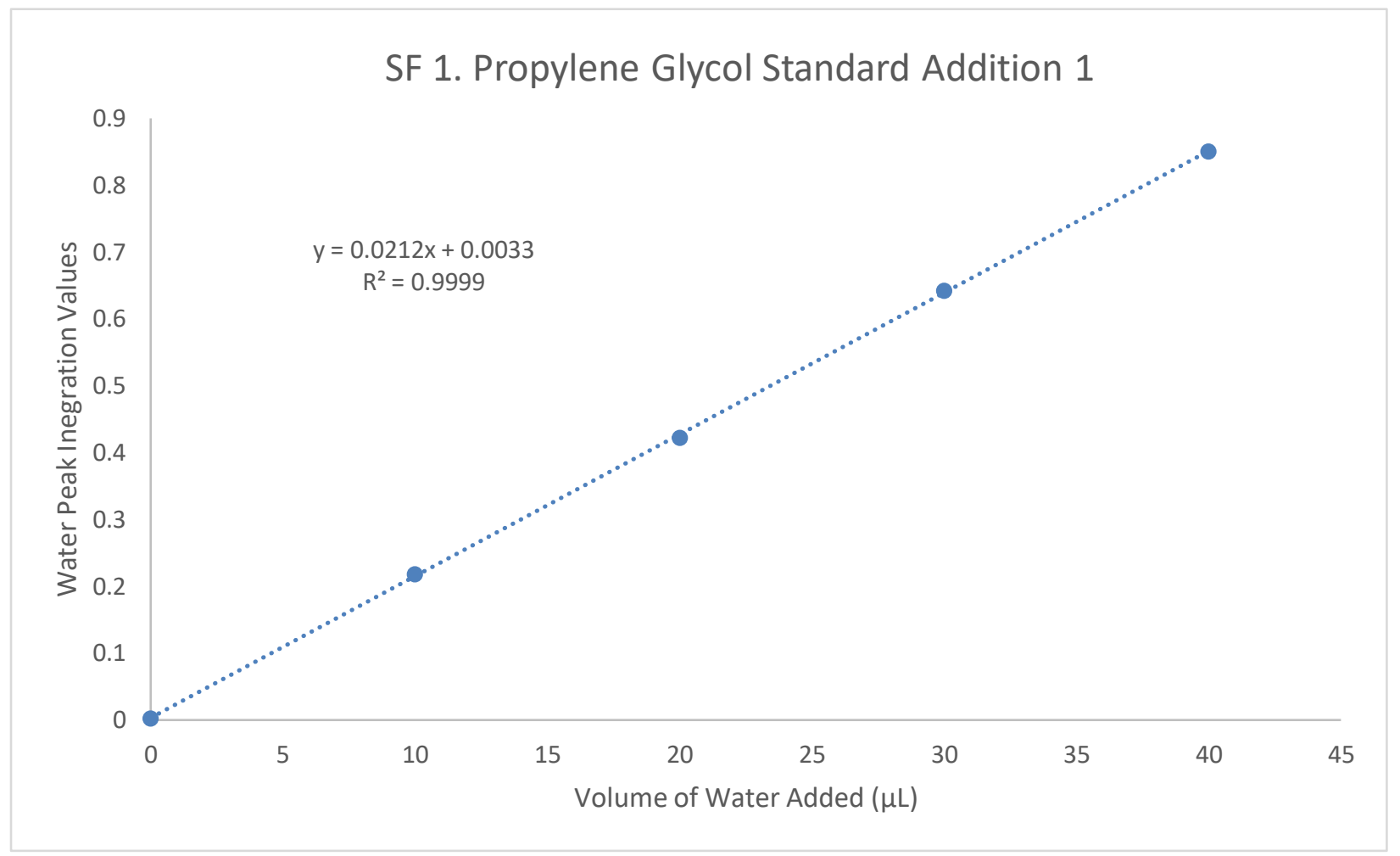

SF 1. Graph of water volume added to the propylene glycol sample vs the NMR peak integration values.

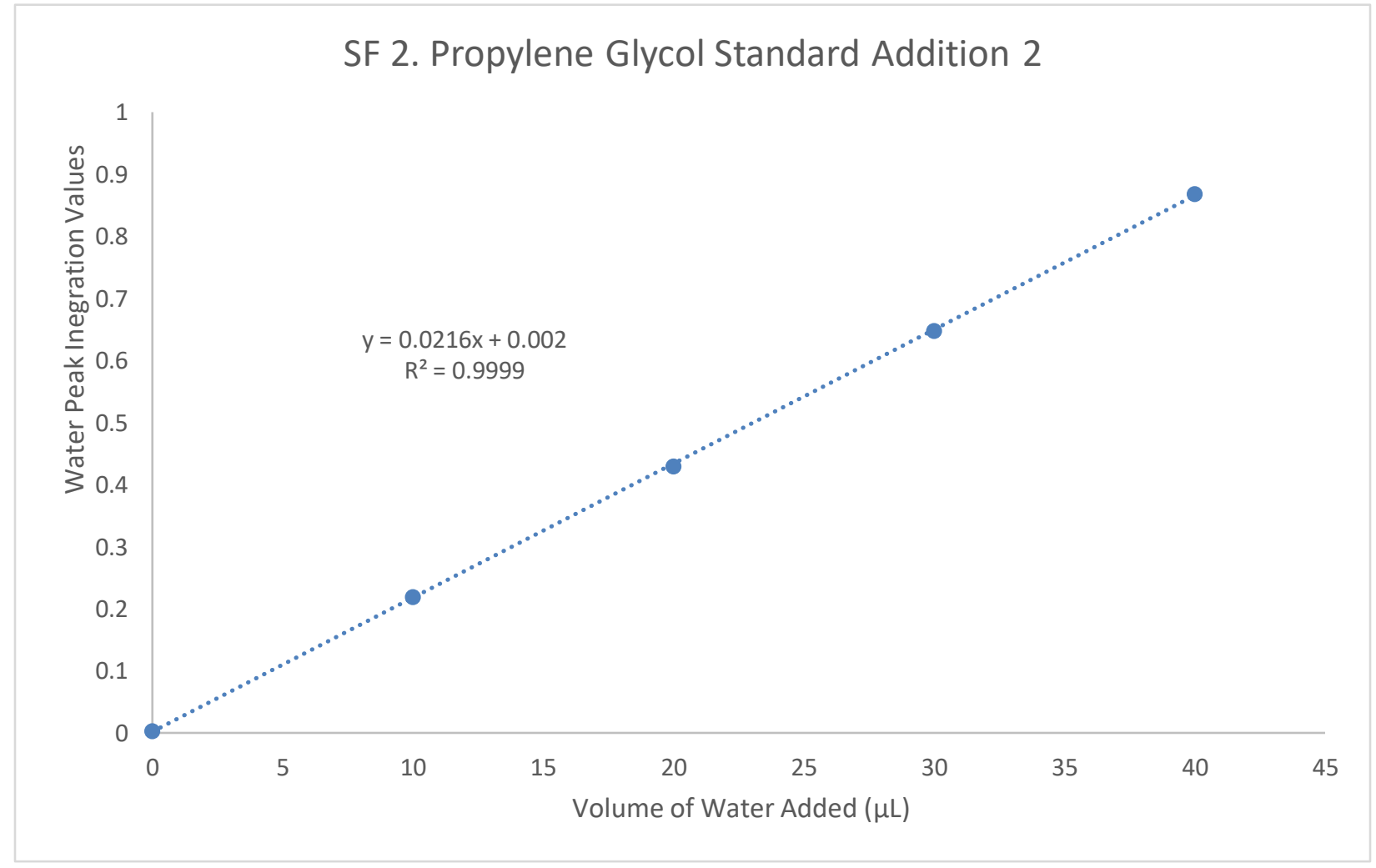

SF 2. Graph of water volume added to the propylene glycol sample vs the NMR peak integration values. 


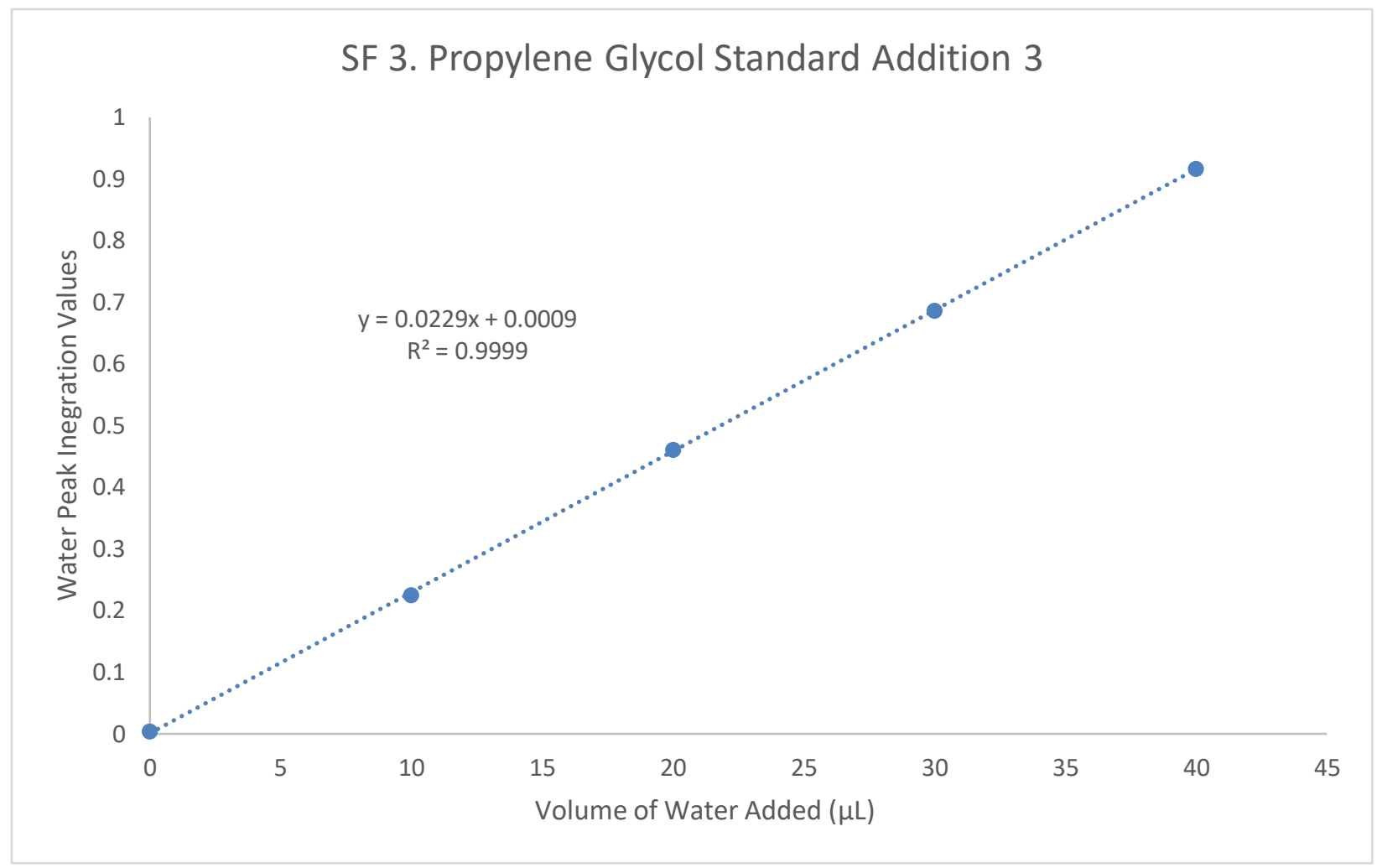

SF 3. Graph of water volume added to the propylene glycol sample vs the NMR peak integration values.

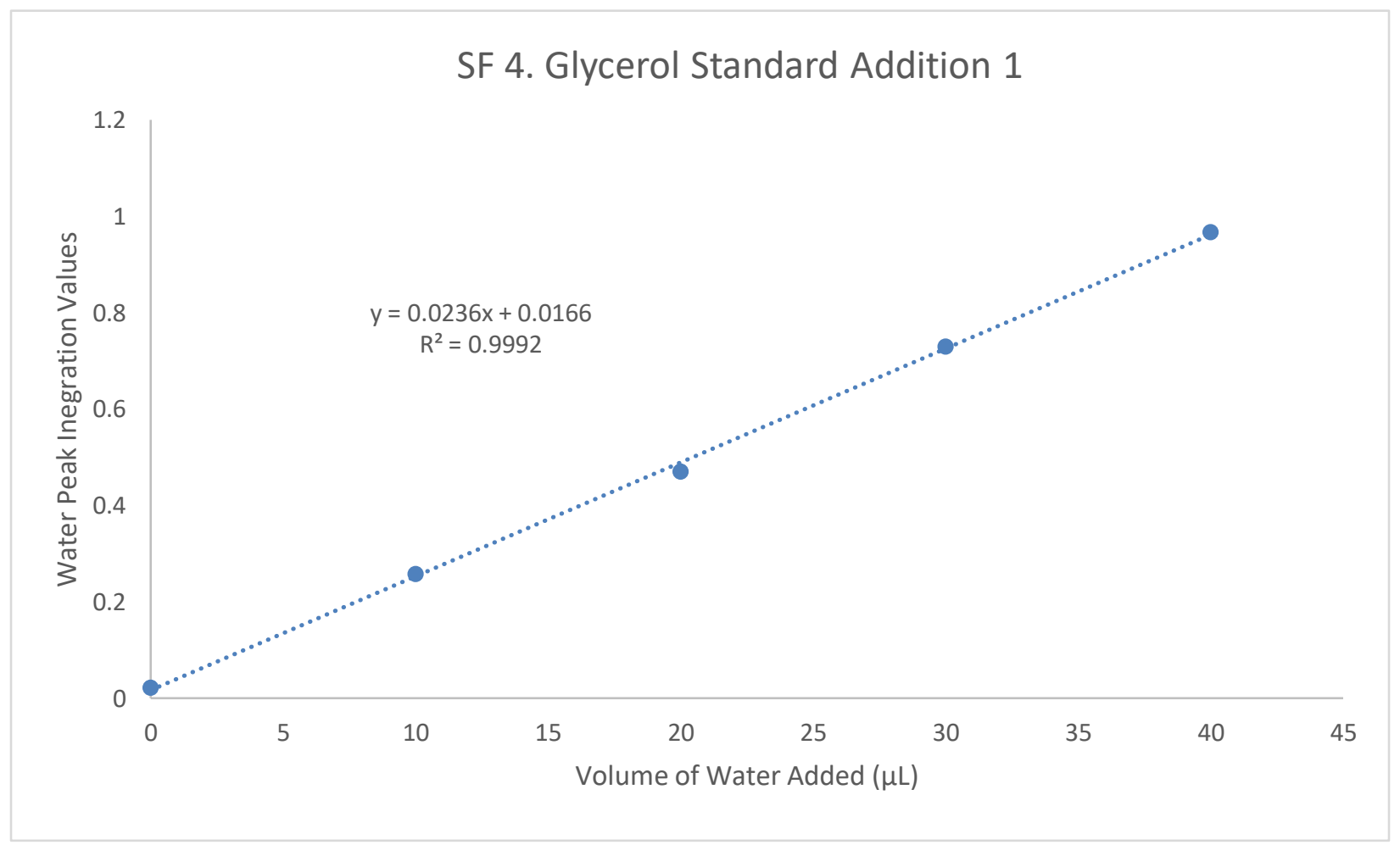

SF 4. Graph of water volume added to the glycerol sample vs the NMR peak integration values. 


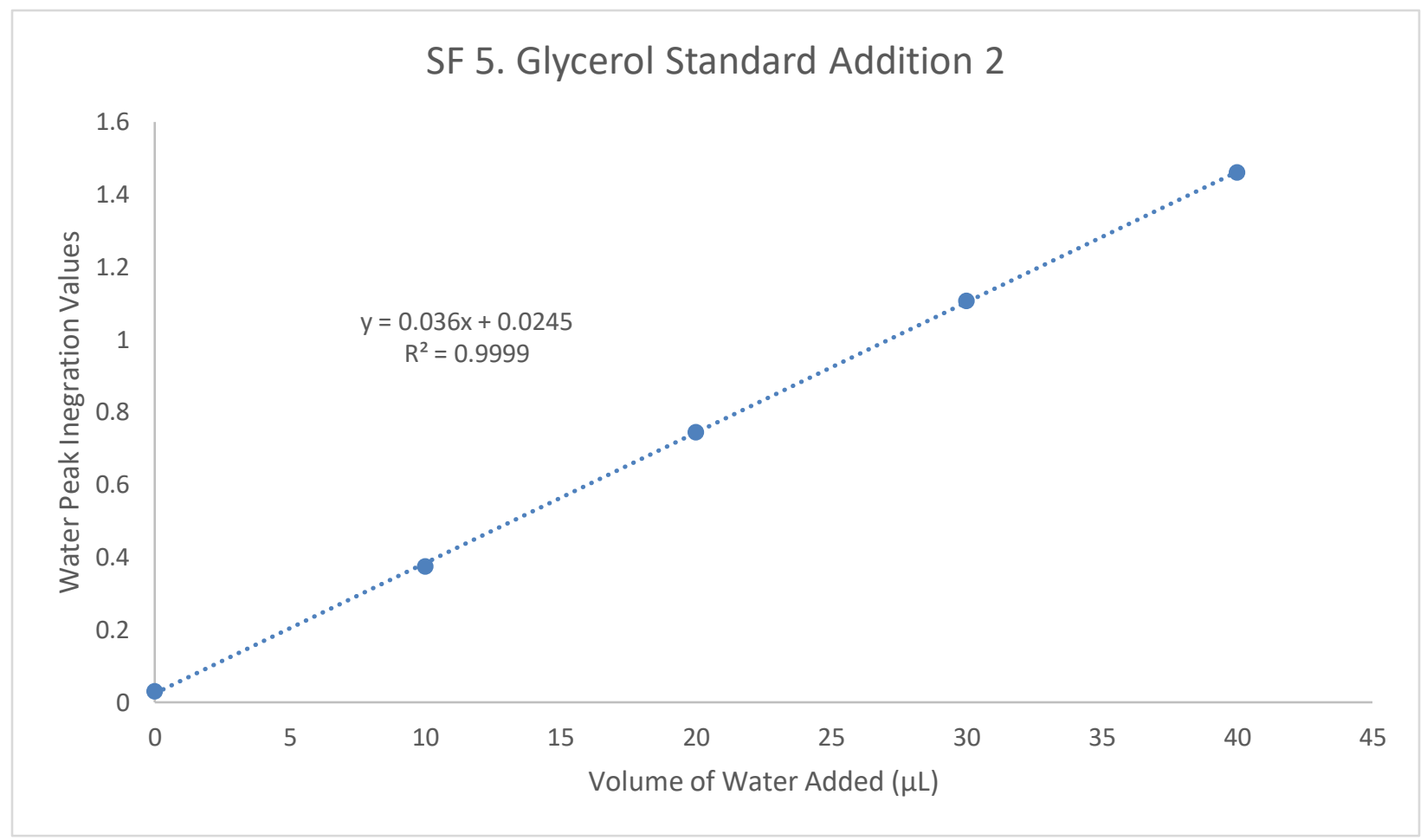

SF 5. Graph of water volume added to the glycerol sample vs the NMR peak integration values.

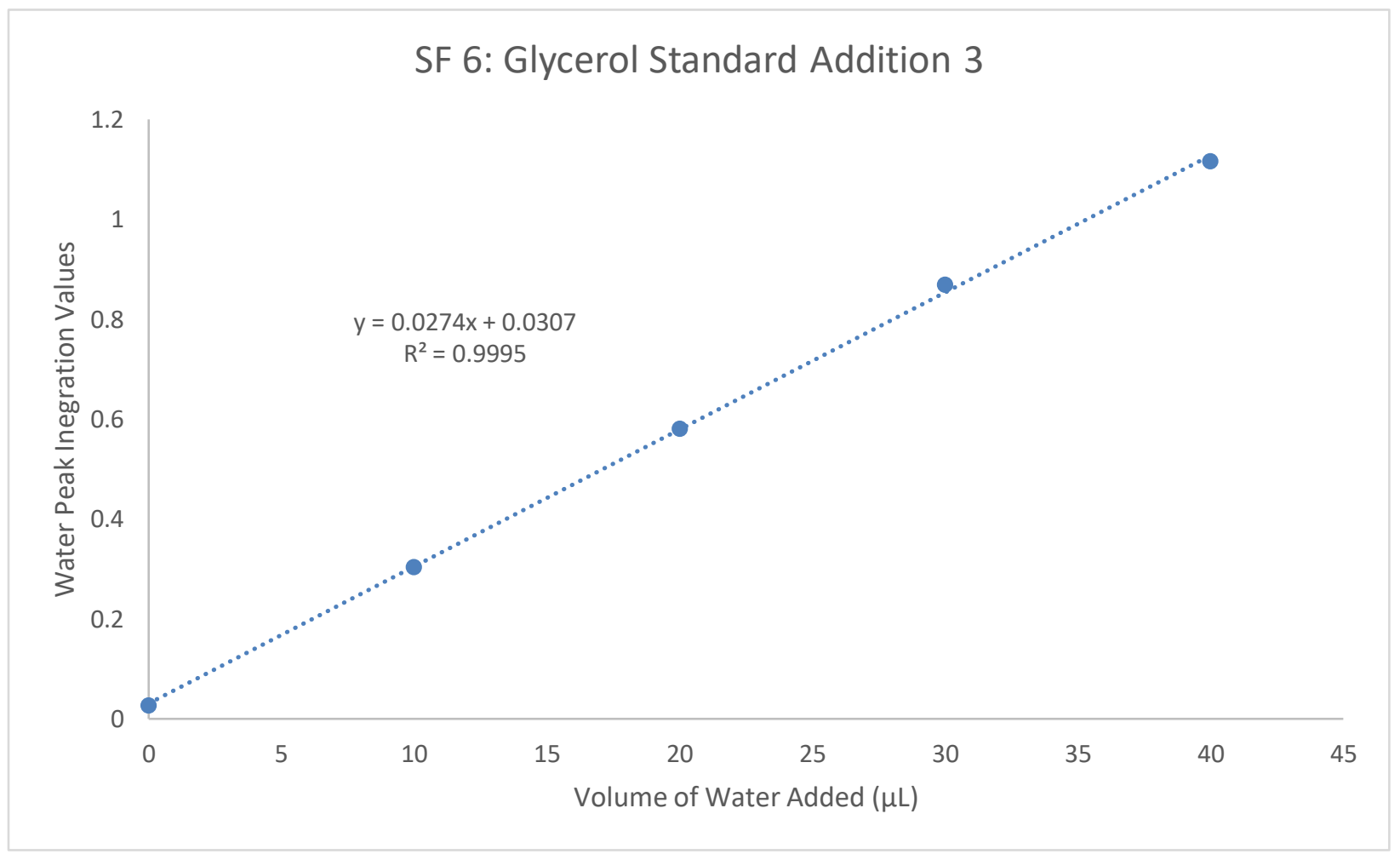

SF 6. Graph of water volume added to the glycerol sample vs the NMR peak integration values. 


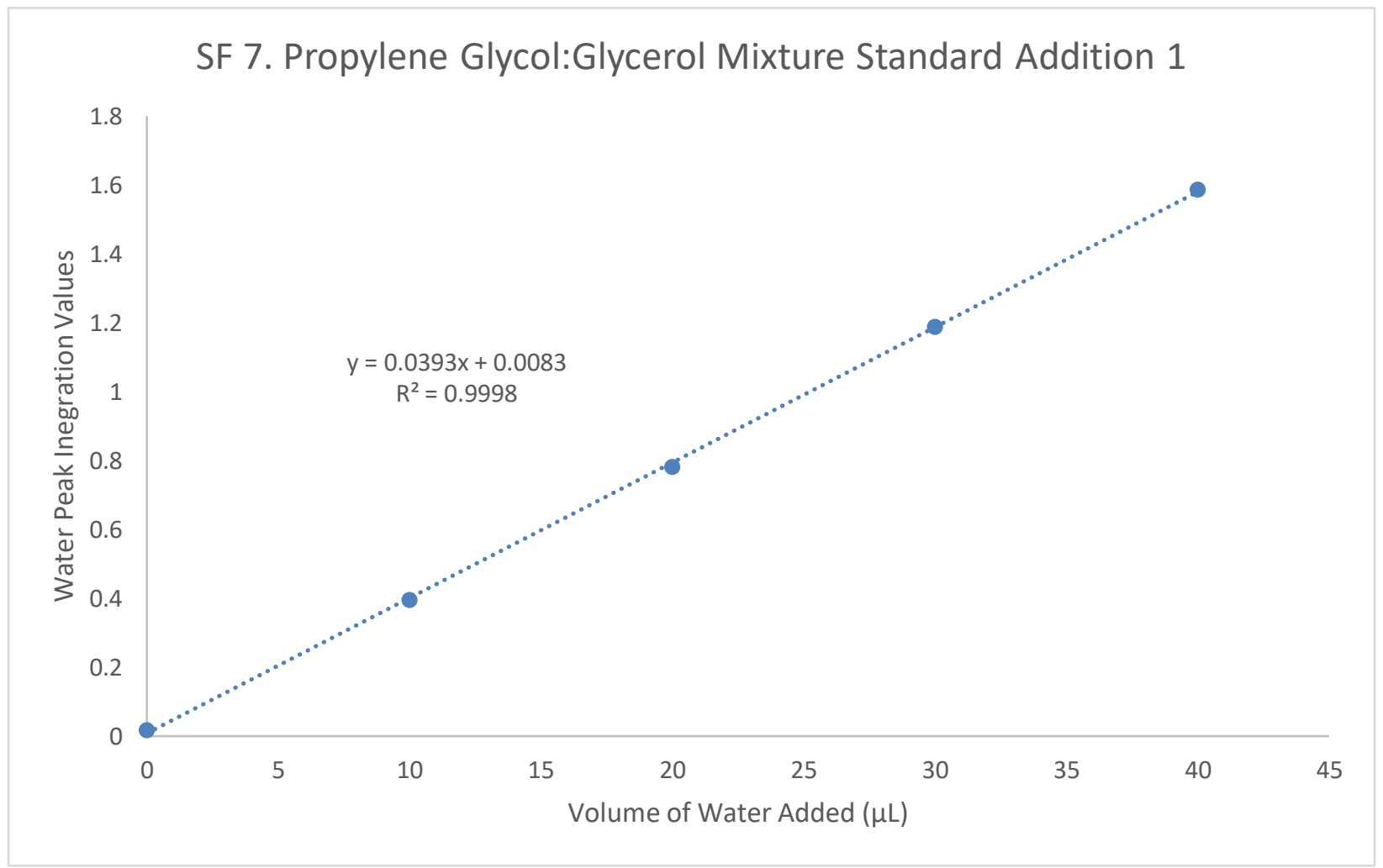

SF 7. Graph of water volume added to the propylene glycol + glycerol sample vs the NMR peak integration values.

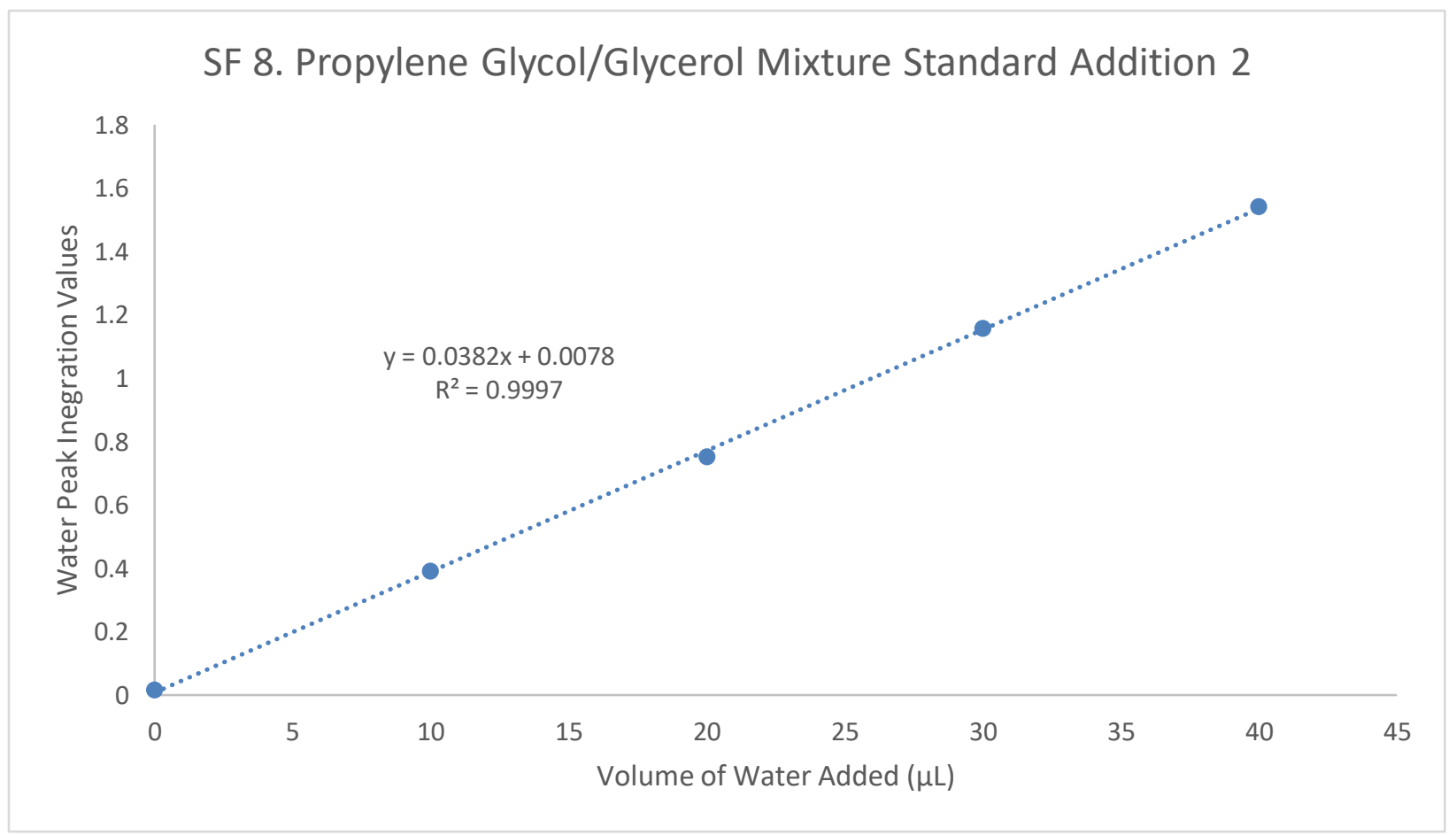

SF 8. Graph of water volume added to the propylene glycol + glycerol sample vs the NMR peak integration values. 


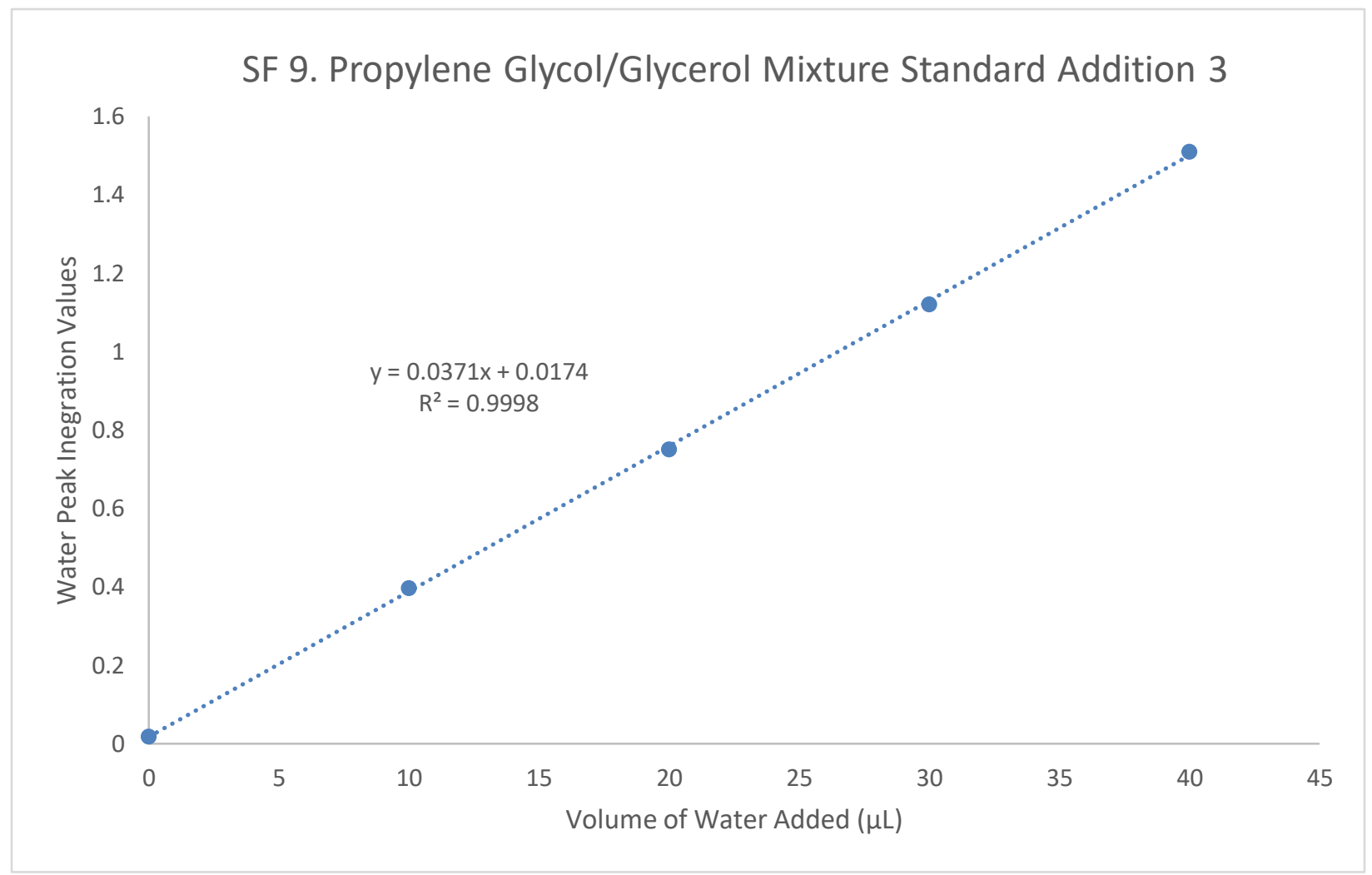

SF 9. Graph of water volume added to the propylene glycol + glycerol sample vs the NMR peak integration values.

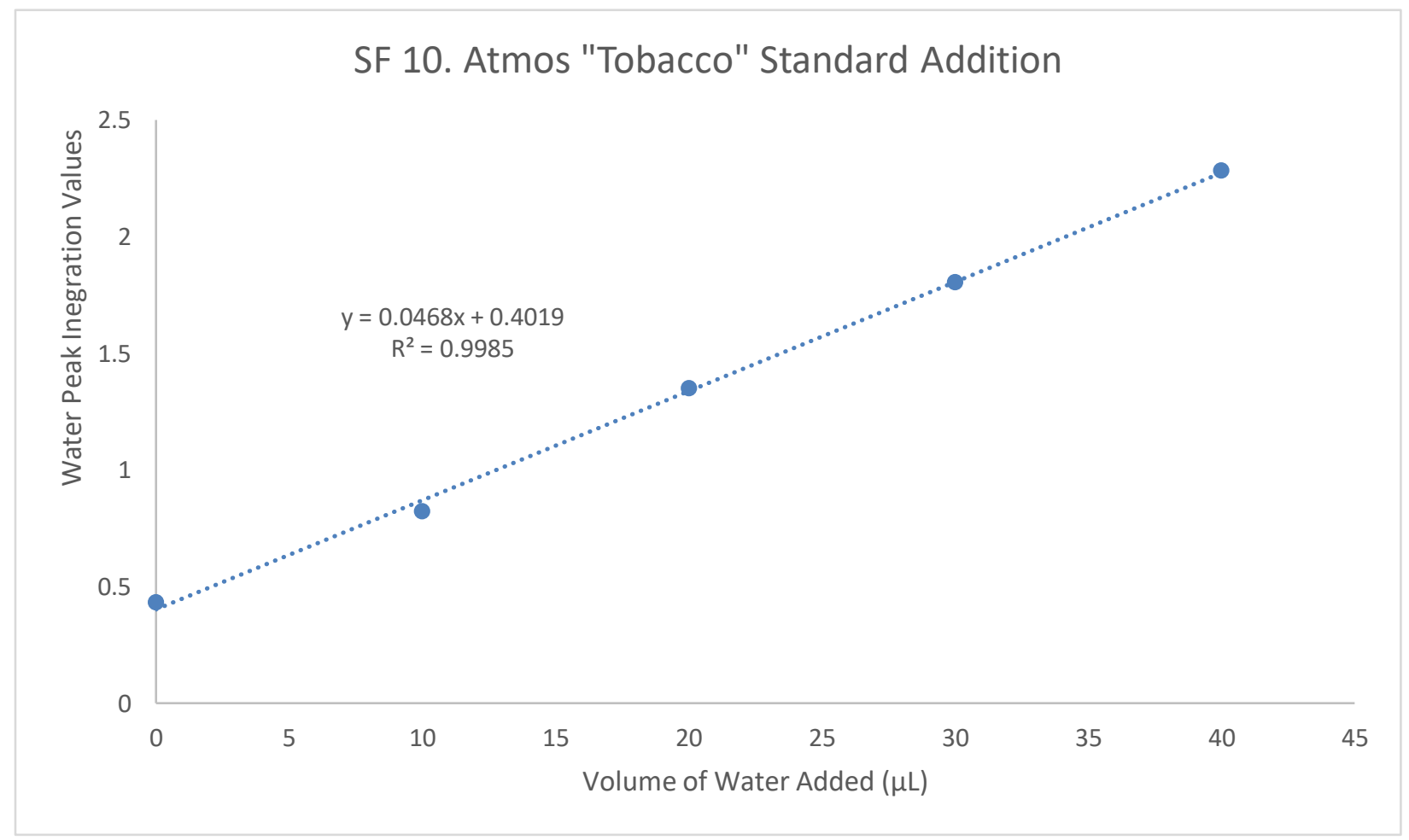

SF 10. Graph of water volume added to the Atmos "Tobacco" sample vs the NMR peak integration values. 


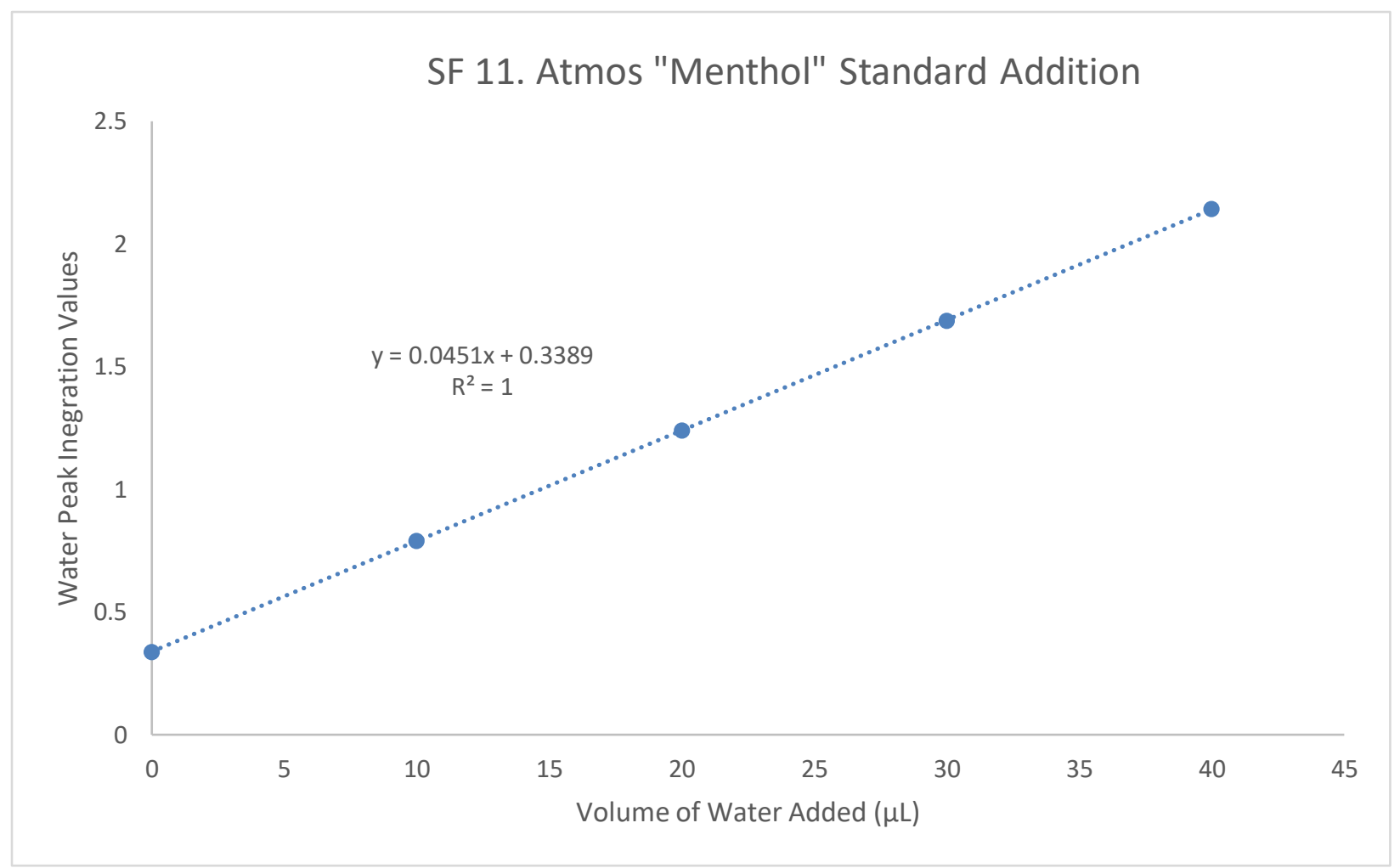

SF 11. Graph of water volume added to the Atmos "Menthol" sample vs the NMR peak integration values.

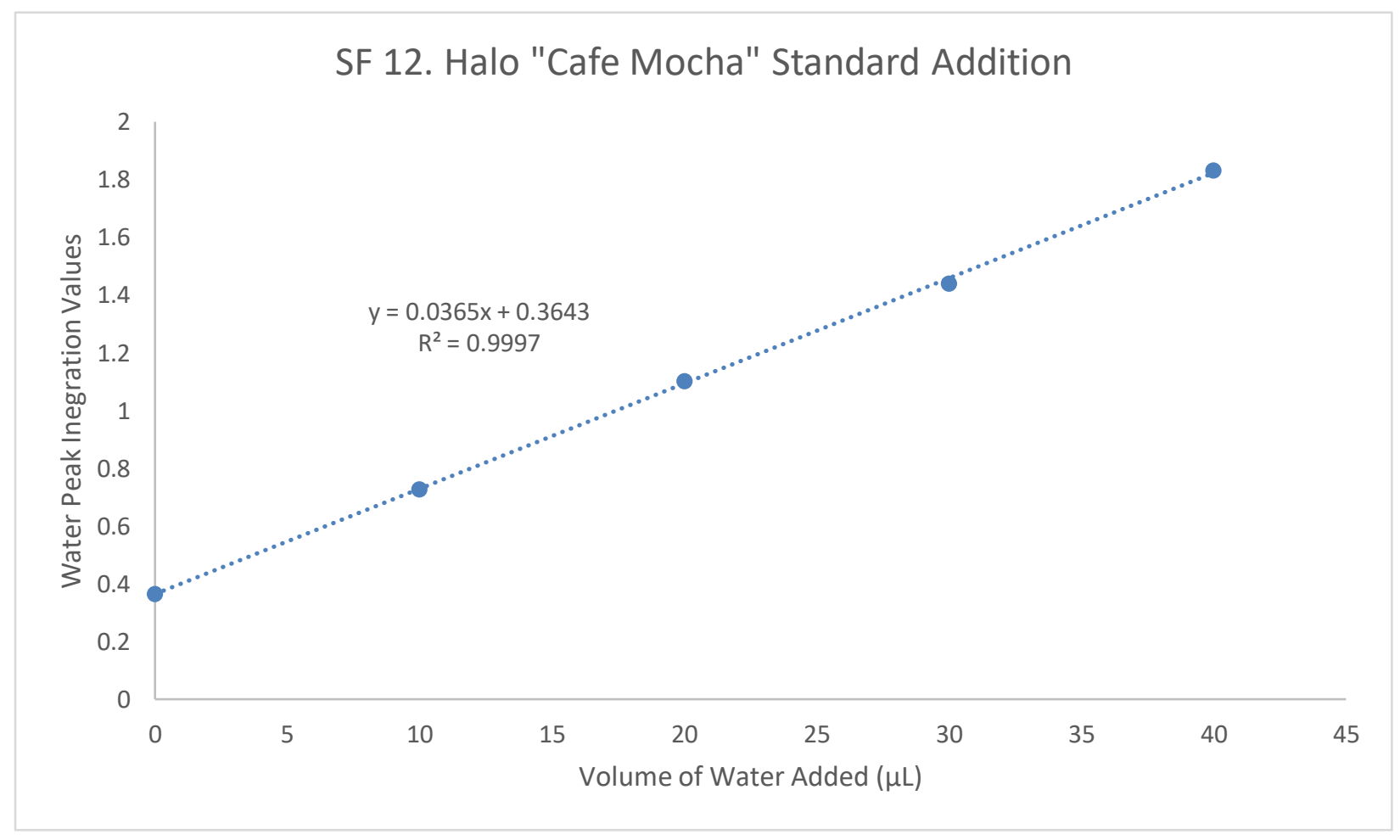

SF 12. Graph of water volume added to the Halo "Café Mocha" sample vs the NMR peak integration values. 